\title{
Sensory processing difficulties (SPD) and their relation to motor performance and to child's perceived competence among children with developmental coordination disorders
}

\begin{abstract}
Objective: Movement is dependent on sensory input. Both Developmental Coordination Disorders (DCD) and sensory processing difficulties (SPD) may reduce child's self-competence and daily function. However, the knowledge about SPD among children with DCD and their relation to their motor performance and selfcompetence is scarce. This study aimed to (1) compare sensory processing abilities and self competence between children with DCD and typical controls (2) examine the relationship between SPD, DCD severity and child's perceived competence among children with DCD.

Methods: The study included 90 children aged 4-10 years: 32 children with DCD and 58 typical controls. All children performed the Movement Assessment Battery for children (M-ABC). Those who scored lower than the $15 \%$ on the $\mathrm{M}-\mathrm{ABC}$ were included in the study group. The parents of all children completed the Short Sensory Profile (SSP) which evaluates the child's sensory processing abilities in daily life. Children with DCD also completed the Pictorial Scale of Perceived Competence and Acceptance for Young Children.

Results: One third of the DCD group showed SPD as compared to $0 \%$ of the controls $\left(\chi^{2} 2=29.11 ; p<.0001\right)$. Among children with DCD, SPD severity correlated with lower balance and manual dexterity. Manual dexterity predicted $32 \%$ of child's physical competence. Auditory/visual sensitivity added $17 \%$ to this prediction.

Conclusion: SPD may be prevalent among children with DCD and partially explain their motor difficulties. However, motor difficulties seem to have a greater impact on self-competence of children with DCD. Further studies with larger samples should examine the relationship between DCD, SPD and self competence. SPD and self competence should be included in the intervention for children with DCD.
\end{abstract}

Keywords: sensory processing, sensory profile, motor performance, selfcompetence, Developmental Coordination Disorders, child development
Volume 3 Issue 2 - 2018

\author{
Batya Engel-Yeger, Dalit Segal \\ Department of Occupational Therapy, Faculty of Welfare and \\ Health Sciences, University of Haifa, Israel
}

Correspondence: Batya Engel-Yeger, Department of Occupational Therapy, Faculty of Welfare and Health Sciences, University of Haifa, Israel,Tel+972-4-8288389,

Email bengel@univ.haifa.ac.il

Received: February 18, 2018 | Published: March 08, 2018

\section{Literature review}

Developmental coordination disorders (DCD) affect approximately $6 \%$ of school aged children and significantly impair their activities of daily living and their academic performance. These impairments are not explicable by age, IQ or neurological condition. ${ }^{1,2}$ Some studies suggest that deficits in motor coordination among children with DCD could be related to sensory processing difficulties (SPD) affecting their motor acuity and their perceptual processes. ${ }^{3,4}$

The phenomenon of SPD is known to be prevalent among 5-15\% of the population. ${ }^{5}$ SPD represent the inability of the central nervous system to modulate the degree, intensity, and nature of responses to sensory input. Thus, SPD may result in sensory hyper or hypo sensitivity. ${ }^{6}$ Individuals with hypersensitivity have low neurological threshold to sensation and thus respond to sensation faster, with more intensity for a longer duration. ${ }^{7}$ They may experience non aversive stimuli as painful and thus express aggressive behaviours, withdrawal, irritability, moodiness, and poor socialization. On the other hand, individuals with sensory hyposensitivity have high neurological threshold to sensation and thus experience difficulties in detecting incoming sensory information. They are described as unmotivated or self-absorbed. Some people with hyposensitivity counter act to their high neurological threshold and actively seek for rich sensory stimuli - they enjoy rich sensory environment and actively initiate behaviours that create sensations. ${ }^{8}$

Studies that focused on SPD among children with DCD referred to specific modalities - mainly to the visual and to the vestibular sensations. Wilson \& McKenzie $^{9}$ highlighted that visual-spatial processing; kinaesthetic perception and cross-modal integration in DCD may be impaired and affect children's movement performance. Indeed, visual-spatial processing and visual-kinesthetic integration which are prerequisites for motor control and stability are impaired 
among $73-87 \%$ of children with DCD. ${ }^{10}$ some studies suggest that specific brain areas that integrate sensory information are less activated among children with DCD. For example, Kashiwagi et al. ${ }^{11}$ argued that lower activation of the left posterior parietal cortex which integrates multimodal sensory information may lead to visual-motor deficits.

In addition to difficulties in motor performance, children with DCD, similar to children with SPD, frequently deal with emotional and cognitive burdens that may impair their function in daily life, lead to difficulties in self-maintenance, as well as to reduced academic achievement and to social isolation. ${ }^{12-14}$ These negative experiences may lead to lower self efficacy and competence. ${ }^{15}$

In middle childhood, children have domain-specific evaluations of their competence or adequacy in different aspects, for example, in regard to their scholastic, social, athletic competence and physical appearance, which contribute to their overall sense of global selfworth. ${ }^{16,17}$ Studies show that children with DCD, even as young as five years old, frequently report that they do not feel competent in performing daily activities. ${ }^{18}$ Engel-Yeger \& Kasis $^{15}$ found that children with DCD aged 5-9 reported significantly lower self efficacy in regard to self-care, school/productivity and leisure activities than typical peers.

The literature stresses the necessity to consider possible factors involved in DCD pathogenesis and their impacts on child's wellbeing. Yet, the existing knowledge about SPD in DCD is limited. Most studies measured sensory processing in specific sensory systems using laboratory settings. More information is needed regarding the prevalence of SPD among children with DCD, as expressed in all sensory modalities and in daily life scenarios. Moreover, knowledge about the interaction between movement performance, SPD and child's self-efficacy and competence should be elaborated. Based on the above, the aims of the present study were: (1) To profile sensory processing difficulties (SPD) of children with DCD and to compare SPD prevalence between children with DCD and typical controls (2) To compare children's perceived self competence and social acceptance between children with DCD and typical controls (3) To examine the relationship between SPD severity, DCD severity and child's perceived competence and acceptance among the DCD group. (4) To examine the ability of SPD to predict motor performance and also examine the contribution of SPD and motor performance to the prediction of self competence among the DCD group.

\section{Methods}

\section{Participants}

Participants were 90 children, aged -10.30 years. All children studied in mainstream public kindergartens and schools. Children's familial socioeconomic status ranged from low to high, in accordance with parents' reports about their mean income level per month and the values published by the Central Bureau for Statistics in Israel. ${ }^{19}$ Table 1 summarizes the socio-demographic information of both groups.

The study group included 32 children with DCD, as diagnosed by a pediatrician/developmental neurologist as suffering from DCD, according to the DSM-V criteria, and by occupational therapists who examined the children by using the Movement Assessment Battery for Children (MABC) [20]. All the children in the study group scored below the 15th percentile in the MABC, indicating their risk for motor difficulties. The control group included 58 children with typical development, who scored above the $15^{\text {th }}$ percentile on the MABC. The description of scores and differences between groups is presented in Table 2.

Table I Participants' socio-demographic data

\begin{tabular}{|c|c|c|c|c|}
\hline & & & $\begin{array}{l}\text { Children } \\
\text { with DCD } \\
(n=32)\end{array}$ & $\begin{array}{l}\text { Typical } \\
\text { controls } \\
(n=58)\end{array}$ \\
\hline \multirow{2}{*}{\multicolumn{2}{|c|}{ Gender }} & $\begin{array}{l}\text { Number of } \\
\text { boys }\end{array}$ & 20 & 31 \\
\hline & & $\begin{array}{l}\text { Number of } \\
\text { girls }\end{array}$ & 12 & 27 \\
\hline \multicolumn{3}{|c|}{ Child's mean age } & $5.69 \pm I .4 I$ & $6.09 \pm 1.83$ \\
\hline \multicolumn{3}{|c|}{ Mother's years of education } & $15.9 \mid \pm 2.27$ & $16.28 \pm 2.34$ \\
\hline \multirow{4}{*}{$\begin{array}{l}\text { Familial } \\
\text { Socioeconomic } \\
\text { status (\%) }\end{array}$} & & & & \\
\hline & Low & & 12.5 & 6.9 \\
\hline & Average & & 31.3 & 58.6 \\
\hline & High & & 56.3 & 34.5 \\
\hline
\end{tabular}

Table 2 Comparison of MABC scores between groups (lower scores = better performance)

\begin{tabular}{|c|c|c|c|c|c|}
\hline & \multicolumn{2}{|c|}{$\begin{array}{l}\text { Children with } \\
\text { DCD } \\
(n=32)\end{array}$} & \multicolumn{2}{|c|}{$\begin{array}{l}\text { Typical controls } \\
(n=58)\end{array}$} & \\
\hline & Mean & SD & Mean & SD & \\
\hline $\begin{array}{l}\text { Mean of } \\
\text { Manual } \\
\text { Dexterity } \\
\text { Total Score }\end{array}$ & 8.01 & 2.58 & 2.66 & 2.59 & $\mathrm{~F}_{1,87}=84.04 * * *$ \\
\hline $\begin{array}{l}\text { Mean of Ball } \\
\text { Skills Total } \\
\text { Score }\end{array}$ & 2.86 & 2.37 & .79 & 1.56 & $\mathrm{~F}_{1,87}=22.93 * * *$ \\
\hline $\begin{array}{l}\text { Mean of } \\
\text { Balance } \\
\text { Total Score }\end{array}$ & 3.64 & 2.46 & .47 & .56 & $\mathrm{~F}_{1,87}=86.94 * * *$ \\
\hline $\begin{array}{l}\text { Total MABC } \\
\text { score }\end{array}$ & 14.93 & 4.01 & 4.31 & 2.77 & $\mathrm{t}_{88}=-14.72 * * *$ \\
\hline $\begin{array}{l}\text { MABC } \\
\text { percentile }\end{array}$ & 7.25 & 4.05 & 50.79 & 24.76 & $\mathrm{t}_{88}=9.85 * * *$ \\
\hline
\end{tabular}

$* * * \mathrm{p} \leq .001$

A significant difference between the groups was found in familial socioeconomic status $\left(\chi^{2}=6.31 ; \mathrm{p}=.04\right)$. Exclusion criteria were positive neurological findings, chronic diseases and syndromes, unfixed sensory impairments (such as glasses for visual impairments), or treatment with medications that affect the functioning of the nervous system.

\section{Instruments}

i. Demographic questionnaire: This questionnaire was designed by the authors and included data on family socio-demographic status, child's health status, medications, treatments, and para-medical therapies. The questionnaire was filled by the children's parents. 
ii. Movement assessment battery for children (MABC) ${ }^{20}$ : The MABC provides an indication of motor functioning across fine and gross motor tasks for children 4-12 years old. There are four age-related item sets, which measure manual dexterity, ball skills, static balance, and dynamic balance. Each set consists of eight items and scores range from 0 to 5 on each item, resulting in a total score between 0 to 40 per set. The MABC has good psychometric properties..$^{20}$

iii. The short sensory profile (SSP) $)^{21}$ : This is a shorter version of the Sensory, ${ }^{8}$ an instrument used for evaluating sensory processing abilities. The SSP is based on the report of the child's main caregiver. It is comprised of the 38 items that demonstrated the highest discriminative power of atypical sensory processing among all the items from the longer version of the Sensory Profile. The seven sections of the SSP found in a normative sample are Tactile Sensitivity; Test/Smell Sensitivity; Movement Sensitivity; Underresponsive/Seeks Sensation; Auditory Filtering; Low Energy/ Weak; and Visual/Auditory Sensitivity. Internal consistency of the sections within the scale ranged from .70 to .90 [8]. Internal validity correlations for the sections ranged from .25 to .76 and were all significant at $\mathrm{p}<.01$. Initial studies of the validity of the SSP demonstrated discriminate validity of $>95 \%$ in identifying children with and without sensory modulation difficulties. ${ }^{21}$ Items are scored on a five-point scale. Both section scores and a total score are recorded on the SSP. The possible range of raw scores on the total scale is 38 to 190 , with higher scores (155-190) reflecting normal performance. A score of 142-154 reflects a probable difference in performance, while a score of 38-141 reflects a definite difference in performance. ${ }^{8}$

iv. The pictorial scale of perceived competence and social acceptance for young children (PSPC) ${ }^{22}$ :This assessment was designed for children in kindergarten and $1^{\text {st }}-2^{\text {nd }}$ classes. It evaluates Cognitive Competence, Physical Competence, Peer Acceptance, and Maternal Acceptance. Each of the four subscales is comprised of six items, constituting a total of 24 items presented in a booklet of pictures of children performing activities related to the different subscales. When presenting each picture, the respondent is read a brief statement about the child in the picture; for example: "This child is good at doing puzzles and this child isn't very good". The respondent is first asked to pick the child in the picture that is mostly like him/her, and then to indicate whether that child is a lot like him/her or just a little like him/her. The child's choices are coded in the form of a score from 1(low competence) to 4 (high competence). Mean score is calculated for each subscale.

\section{Procedure}

Ethical approval for this study was provided by the Israeli Ministry of Education. All parents signed a consent form allowing their children to participate in the study and completed the demographic questionnaire. A meeting was set with all children who fulfilled the inclusion criteria in their homes (control group) or at the clinic (study group). All evaluations were administered to each child individually in a quiet room while the parents completed the SSP.

\section{Data analysis}

Descriptive statistics were used to describe the sample and the main variables. T-test and Chi square test analyzed the differences between the groups in regard to socio-demographic parameters, in their dominant hand and in their sensory performance range distribution. MANCOVA with familial socio-economic status serving as covariate was performed to examine the significance of the differences between groups in regard to the dependent variables. The differences between the groups in the total scores of the MABC and SSP were analyzed by t-test. Chi square analysis examined whether differences exist between groups in the percentage of children found in each of the sensory level of performance.

Partial correlation test examined the significance of correlations between the dependent variables among the DCD group with familial socio-economic status serving as covariate. A linear regression analysis was applied to test the relative contribution of group membership and SPD to the prediction of motor performance and self competence. The level of significance was set at .05 for all statistical tests.

\section{Results}

\section{Sensory processing abilities of children with DCD as compared to typical controls}

Significant differences were found between both groups in all SSP scales-children with DCD showed elevated sensitivity and greater tendency of sensory seeking/under responsiveness than the typical control (Table 3).

A significant greater prevalence of movement sensitivity, underresponsiveness/sensation seeking and low energy was found among children with DCD as compared to the control group - a significant higher percentage of children with DCD was found in the "definite difference level" of these SSP subscales (Table 4).

Table 3 Comparison of SSP sum-scores between both groups

\begin{tabular}{|c|c|c|c|c|c|c|c|}
\hline \multirow[b]{2}{*}{ SSP subtest } & \multicolumn{3}{|c|}{$\operatorname{DCD}(n=32)$} & \multicolumn{4}{|c|}{ Controls $(n=58)$} \\
\hline & Mean & SD & Range & Mean & SD & Range & \\
\hline Tactile sensitivity & 30.22 & 4.21 & $17-35$ & 32.39 & 2.78 & $23-35$ & $\mathrm{~F}_{1,87}=8.57 * *$ \\
\hline Taste/smell sensitivity & 16.34 & 3.91 & $7-20$ & 17.72 & 2.64 & $10-20$ & $\mathrm{~F}_{1,87}=3.87 *$ \\
\hline Under responsive/seek & 26.84 & 5.41 & $15-35$ & 31.77 & 3.58 & $21-35$ & $\mathrm{~F}_{1,87}=25.62 * * *$ \\
\hline Auditory filtering & 25.12 & 3.82 & $13-30$ & 27.53 & 2.37 & $21-30$ & $\mathrm{~F}_{1,87}=13.29 * * *$ \\
\hline Low energy/ weak & 23.34 & 4.69 & $11-30$ & 28.27 & 2.27 & $23-30$ & $\mathrm{~F}_{1,87}=42.67 * * *$ \\
\hline Total & 153.84 & 23.46 & $83-190$ & I75.II & 11.97 & $\begin{array}{l}147- \\
190\end{array}$ & $\mathrm{t}_{88}=5.67^{* * *}$ \\
\hline
\end{tabular}

$* \mathrm{p} \leq .05 ; * * \mathrm{p} \leq .01 ; * * * \mathrm{p} \leq .001$ 
Table 4 The percentage of children from each group in each sensory level of performance

\begin{tabular}{llllllll}
\hline & \multicolumn{2}{l}{ Typical performance } & & \multicolumn{2}{l}{ Probable difference } & \multicolumn{4}{l}{ Definite difference } \\
\cline { 2 - 8 } SSP subtest & DCD & controls & DCD & controls & DCD & controls & $\chi^{2}$ \\
\hline Tactile sensitivity & 62.5 & 84.5 & 25 & 12.1 & 12.5 & 3.4 & $5.9 I^{*}$ \\
Taste/smell sensitivity & 65.6 & 86.2 & 12.5 & 8.6 & 21.9 & 5.2 & $6.59^{*}$ \\
Movement sensitivity & 59.4 & 79.3 & 9.4 & 15.5 & 31.3 & 5.2 & $11.43^{* *}$ \\
Underresponsive/seek & 50 & 89.7 & 15.6 & 8.6 & 34.4 & 1.7 & $21.63^{* * *}$ \\
Auditory filtering & 81.3 & 96.6 & 12.5 & 3.4 & 6.3 & 0 & $6.69 *$ \\
Low energy/weak & 37.5 & 81 & 9.4 & 17.2 & 53.1 & 1.7 & $34.08^{* * *}$ \\
Visual/auditory sensitivity & 62.5 & 96.6 & 21.9 & 3.4 & 15.6 & 0 & $18.89 * * *$ \\
Total & 53.1 & 98.3 & 18.8 & 1.7 & 28.1 & 0 & $29.11 * * *$ \\
\hline
\end{tabular}

$\mathrm{p} \leq 0.05 * ; \mathrm{p} \leq 0.0 I^{* * ;} \mathrm{p} \leq 0.00 I^{* * *}$

\section{Children's perceived self competence and social acceptance of children with DCD as compared to typical controls}

No significant difference was found between both groups in their PSPC scores. However, children with DCD reported lower perceived competence than the controls in all PSCS subscales except for Maternal Acceptance. The difference between groups was significant when comparing specific items of the PSPC in which children with DCD reported significant lower scores: "Good at counting" (for kindergartens)/" Can write words" (for school aged children) (related to the cognitive subscale) (mean typical=4.01 $1.01 ;$ mean DCD=3.73 $\pm .54 ; \mathrm{F}=6.35, \mathrm{p}=.001$ );" Good at skipping" (related to the physical subscale) (mean typical=3.92 \pm .38 , mean $\mathrm{DCD}=3.47 \pm 1.08 ; \mathrm{F}=3.96, \mathrm{p}=.05$ ) and "Eats dinner at friends' house (for kindergartens)/" others sit next to you (for school aged children) (related to the peer acceptance subscale) (mean typical=3.62 \pm .83 ; mean $\mathrm{DCD}=3.04 \pm 1.18 ; \mathrm{F}=4.06, \mathrm{p}=.05)$. Figure 1 depicts the comparison of PSPC scores between both groups.

\section{The relationship between motor performance, sensory processing and child's perceived competencel acceptance among the DCD group}

When examining the correlations between MABC and SSP scores among the DCD group, lower manual dexterity significantly correlated with elevated movement sensitivity $(\mathrm{r}=-.43, \mathrm{p}=.01)$, greater under-responsiveness/sensation seeking $(\mathrm{r}=-.37, \mathrm{p}=.04)$ ( represent atypical sensory processing and elevated MABC scores represent worse motor performance, the correlations are marked in minus). Lower balance skills significantly correlated with lower energy level $(\mathrm{r}=-.47, \mathrm{p}=.008)$ and elevated auditory/visual sensitivity $(\mathrm{r}=-.52, \mathrm{p}=.003)$.

The correlations between PSPC, MABC and SSP scores among the DCD group revealed that reduced physical acceptance significantly correlated with lower manual dexterity $(\mathrm{r}=.56, \mathrm{p}=.006)$ and with elevated auditory/visual sensitivity $(\mathrm{r}=.46, \mathrm{p}=.003)$.

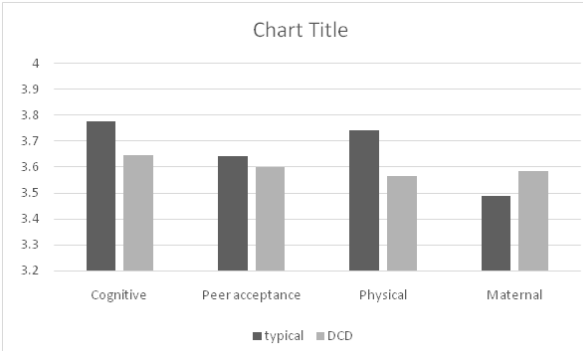

Figure I Comparing Perceived Competence and Social Acceptance between groups.

The ability of SPD to the predict motor performance and the contribution of SPD and motor performance to the prediction of self competence among the DCD group

Elevated movement sensitivity accounted for $18 \%$ of the variance of lower manual dexterity. Low energy added $11 \%$ to this prediction.
Elevated auditory/visual sensitivity accounted for $25 \%$ of the variance in regard to lower balance performance. Elevated movement sensitivity accounted for $17 \%$ of the variance in regard to lower general motor performance as presented by the total MABC score (Table 5).

When referring to PSPC scores-lower manual dexterity accounted for $32 \%$ of the variance in regard lower physical competence while elevated auditory/visual sensitivity added $17 \%$ to this prediction. 
Table 5 Predicting motor performance by groups and sensory processing abilities

\begin{tabular}{|c|c|c|c|c|c|c|}
\hline \multirow[b]{2}{*}{ Variable } & \multicolumn{3}{|c|}{ Model I } & \multicolumn{3}{|c|}{ Model 2} \\
\hline & B & SE B & $\beta$ & B & SE B & $\beta$ \\
\hline \multicolumn{7}{|l|}{ Manual Dexterity } \\
\hline Movement sensitivity & -.34 & .13 & $-.42 *$ & -.53 & .16 & $-.66^{*}$ \\
\hline Low energy & & & & .23 & .11 & $.4 I^{*}$ \\
\hline $\mathrm{R}^{2}$ & 18 & & & 29 & & \\
\hline$F_{1,31}$ for change in $R^{2}$ & $6.45^{*}$ & & & $4.68^{*}$ & & \\
\hline \multicolumn{7}{|l|}{ Balance } \\
\hline Auditory/visual sensitivity & -.26 & .08 & $-.50 * *$ & & & \\
\hline $\mathrm{R}^{2}$ & 25 & & & & & \\
\hline$F_{1,31}$ for change in $R^{2}$ & $10.18^{* *}$ & & & & & \\
\hline \multicolumn{7}{|l|}{ Total MABC score } \\
\hline Movement sensitivity & -.51 & .21 & $-.4 I^{* *}$ & & & \\
\hline $\mathrm{R}^{2}$ & 17 & & & & & \\
\hline $\mathrm{F}_{1,31}$ for change in $\mathrm{R}^{2}$ & $5.99 *$ & & & & & \\
\hline \multicolumn{7}{|l|}{ Physical competence } \\
\hline Manual Dexterity & .12 & .04 & $.56 * *$ & .11 & .03 & $.53 * *$ \\
\hline Movement sensitivity & & & & .05 & .02 & $.49 *$ \\
\hline $\mathrm{R}^{2}$ & 32 & & & 49 & & \\
\hline $\mathrm{FI}, 3 \mathrm{I}$ for change in $\mathrm{R}^{2}$ & $9.92 * *$ & & & $5.78^{*}$ & & \\
\hline
\end{tabular}

$* \mathrm{p} \leq .05 ; * * \mathrm{p} \leq .01$

\section{Discussion}

The present study profiled SPD among children with DCD and examined the relationship between SPD, motor performance and child's perceived self-competence. This study suggests that elevated prevalence of SPD may characterize children with DCD and that SPD may be considered as a possible mechanism involved in DCD. This finding supports previous reports. ${ }^{21-23}$ This involvement may have unique expressions in specific modalities and behaviours and partially explain the motor difficulties of children with DCD. Hence, it is recommended to evaluate the sensory profile of children with $\mathrm{DCD}$, as a complementary to the assessment of child's motor performance. Parents' report regarding SPD expressions in daily life of their child, as reflected by the SSP, may elevate their awareness to the possible involvement of SPD in DCD regarding daily functional aspects.

In the present study, SPD unique expressions were extremely emphasized in relation to greater movement sensitivity, lower energy level (which represents insufficient proprioception processing) and under-responsiveness/sensation seeking, all of which are known to be significantly associated with movement control. ${ }^{24}$ This association may provide a possible explanation for the impaired balance of children with DCD, their lower abilities to regulate muscle activity which is less uniform and consistent ${ }^{25}$ and their difficulties in acquiring motor skills based on the integration of vestibular and proprioceptive information. ${ }^{26}$

Indeed, among the DCD group, movement sensitivity, auditory/ visual sensitivity, lower energy and under-responsiveness/sensation seeking significantly correlated specifically with balance performance and manual dexterity. These results were strengthened by the ability of SPD to predict motor performance among children with DCD. Lower motor performance was significantly predicted by elevated movement sensitivity. Movement sensitivity as well as low energy (difficulties in processing proprioceptive information) significantly predicted lower Manual Dexterity in which acuity is heavily dependent on adequate postural control and proper feedback that comes from the proprioceptive system. Roche et al. ${ }^{27}$ claimed that children with DCD present inaccurate movements since they are either not aware to the sensory information to detect errors or they do not use sensory feedback to correct their motor actions.

The present study also found that reduced balance performance was predicted by elevated auditory/visual sensitivity. This supports previous reports that highlight the significant role of difficulties in the visual and auditory processing to the motor coordination difficulties of individuals with DCD. ${ }^{3,4,13,27,28}$ For example, studies found that deficiencies in auditory/visual processing lead to difficulties in rhythm and timing experienced by children with $\mathrm{DCD},{ }^{29-31}$ as expressed in finger tapping performance of children with DCD which was less accurate and more variable in frequency and coordination. ${ }^{27}$

Hence, SPD may impair the ability to correct errors in position, and to modulate acuity, velocity, and force of movement. ${ }^{23,26,32}$ Problems in the modulation and integration of sensory input coming from the vestibular, proprioception, visual and auditory systems may reduce motor performance, and mainly balance and manual dexterity. ${ }^{33}$ The relationship between deficiencies in sensory processing and impaired motor control due to possible mutual neurodevelopmental underlying mechanisms may be supported by fact that SPD is highly prevalent among other co morbidities of DCD such as ADHD. ${ }^{34}$ Studies highlight the frequent motor impairments of children with ADHD that include insufficient balance control and reduced manual dexterity. ${ }^{35,36}$ 
The sensory and motor difficulties that children with DCD may experience have significant negative implications on child's daily life and self competence. ${ }^{37,38}$ The present study found that children with DCD expressed lower perceived self competence and social acceptance than typical controls. This difference was significant in specific items of the PSPC such as writing, skipping, which require coordination, rhythm and timing, and in items related to social interactions - all of which are known to be impaired among children with DCD. ${ }^{24}$

The present study emphasized not only the relationship between SPD and motor performance, but also the ability of both factors to predict lower self competence of children with DCD. Specifically lower physical competence of the study group was predicted by manual dexterity and by auditory/visual sensitivity. These relationships may support the studies showing that children with DCD report about their lower self competence and efficacy in daily life in which manual dexterity is highly involved in self-care activities and school/ productivity activities mainly in regard to handwriting. ${ }^{37}$ Moreover, the literature emphasized that as worse was the motor performance, the lower was the self efficacy. ${ }^{39}$ Hence, when SPD is coupled with ineffective motor control among children with DCD it may enhance their difficulties in gross motor as well as in fine motor performance, reduce their ability to adaptively function in daily activities ${ }^{25,40,41}$ and to feel competent.

Considering that lower self-competence, lower self-worth and social problems are common among children with DCD and have negative implications on their well being, ${ }^{42}$ further studies on larger samples and in variant age groups should be performed in order to better understand the relationship between these factors. This understanding may significantly contribute to the evaluation and intervention process and elevate child's well being and quality of life. Moreover, the fact that children with DCD are able to report about their lower self competence supports the client cantered approach ${ }^{43}$ and directs clinicians toward an intervention plan which is focused on the child's specific needs. The children's awareness to their difficulties may enhance their involvement in therapy, and lead to better intervention outcomes ${ }^{44}$ and limit social isolation, ${ }^{24}$ academic difficulties and mental problems. ${ }^{15}$

In conclusion, possible commonality may exist between DCD and SPD due to underlying neuro-developmental deficiency. ${ }^{45}$ The specific SPD patterns of children with DCD highlight that DCD evaluation should screen for SPD "red flags". When SPD is presented, it is of most important to understand its relation to motor performance and to child's function and self competence, as expressed in daily environments. Clinicians should elevate child/parents'/teachers' awareness to this relationships. Intervention should include sensory stimuli and sensory-motor integration in order to enhance the development of brain sensory-motor networks and related outcomes such as body scheme and motor control. Referring to co morbidities such as SPD may minimize DCD negative outcomes, reduce avoidance from physical activities; reduce loneliness and solitude and elevate self esteem and competence. ${ }^{39,46}$ In that way we can enhance the child's optimal development and well being.

\section{Acknowledgements}

None.

\section{Conflict of interest}

Authors declare that there are no conflicts of interest.

\section{References}

1. American Psychiatric Association. Diagnostic and Statistical Manual of Mental Disorders. 4th ed. American Psychiatric Press, Washington DC; 2003.

2. Cermak SA, Larkin D. Developmental coordination disorder. Cengage Learning. 2002.

3. Zoia S, Castiello U, Blason L, et al. Reaching in children with and without developmental coordination disorder under normal and perturbed vision. Dev neuropsychol. 2005;27(2):257-273?

4. Van WH, Weerdt DW, De CP, et al. Aspects of the validity of the Movement Assessment Battery for Children. Hum Mov Sci. 2004:23(1):49-60.

5. Simeonsson RJ, Leonardi M, Lollar D, et al. Applying the International Classification of Functioning, Disability and Health (ICF) to measure childhood disability. Disabil Rehabil. 2003;25(11-12):602-610?

6. Dunn W. The Impact of Sensory Processing Abilities on the Daily Lives of Young Children and Their Families: A Conceptual Model. Infants \& Young Children. 1997;9:23-35.

7. Miller LJ, Anzalone ME, Lane SJ, et al. Concept evolution in sensory integration: a proposed nosology for diagnosis. Am J Occup Ther. 2007;61:135-140

8. Dunn W. The Sensory Profile: Examiner's manual. Psychological Corporation, San Antonio, TX;1999.

9. Wilson $\mathrm{PH}, \mathrm{McKenzie} \mathrm{BE}$. Information processing deficits associated with developmental coordination disorder: A meta-analysis of research findings. J Child Psychol Psychiatry. 1998;39(6):829-840.

10. Macnab JJ, Miller LT, Polatajko HJ. The search for subtypes of DCD: Is cluster analysis the answer?. Hum Mov Sci. 2001;20(1-2):49-72.

11. Kashiwagi M, Iwaki S, Narumi Y, et al. Parietal dysfunction in developmental coordination disorder: a functional MRI study. Neuroreport . 2009;20(15):1319-1324.

12. Dewey D, Kaplan BJ, Crawford SG, et al. Developmental coordination disorder: associated problems in attention, learning, and psychosocial adjustment. Hum Mov Sci. 2002;21(5-6):905-918.

13. Schoemaker MM, Wees M, Flapper B, et al. Perceptual skills of children with developmental coordination disorder. Hum Mov Sci. 2001;20(12):111-133.

14. Zwicker JG, Missiuna C, Boyd LA. Neural correlates of developmental coordination disorder: A review of hypotheses. $J$ Child Neurol. 2009;24(10):1273-1281.

15. Engel YB, Hanna KA. The relationship between Developmental Coordination Disorders, child's perceived self-efficacy and preference to participate in daily activities. Child Care Health Dev. 2010;36(5):670-677.

16. Harter S. Processes underlying the construction, maintenance, and enhancement of the self-concept in children. Psychological perspectives on the self. 1986;27(3):7-181.

17. Harter S. Self-perception profile for children: Manual and Questionnaires. Denver, Denver University; 2012.)

18. Dunford C, Missiuna C, Street E, et al. Children's perceptions of the impact of developmental coordination disorder on activities of daily living. The British Journal of Occupational Therapy. 2005;68(5):207-214. 
19. http://www1.cbs.gov.il/reader/cw_usr_view_Folder?ID=141

20. Henderson SE, Sugden DA. The movement assessment battery for children. Psychological Corporation, San Antonio, TX; 1992.

21. McIntosh DN, Miller LJ, Shyu V, et al. Development and validation of the short sensory profile. Sensory profile manual. 1999;59-73.

22. Harter S, Pike R. The pictorial scale of perceived competence and social acceptance for young children. Child development. 1984; 55(6):19691982.]

23. Inder JM, Sullivan SJ. Motor and postural response profiles of four children with developmental coordination disorder. Pediatric Physical Therapy.2005;17(1):18-29.

24. Piek JP, Dyck MJ. Sensory-motor deficits in children with developmental coordination disorder, attention deficit hyperactivity disorder and autistic disorder. Hum Mov Sci. 2004;23(3-4):475-488.

25. Fong SS, Tsang WW, Ng GY. Altered postural control strategies and sensory organization in children with developmental coordination disorder. Hum Mov Sci. 2012;31(5):1317-1327.

26. Tsai CL, Wu SK, Huang CH. Static balance in children with developmental coordination disorder. Hum Mov Sci. 2008;27(1):142-153?

27. Roche R, Wilms FAM, Clark JE, et al. Auditory and visual information do not affect self-paced bilateral finger tapping in children with DCD. Human movement science. 30(3):658-671.

28. Coleman R, Piek JP, Livesey DJ. A longitudinal study of motor ability and kinaesthetic acuity in young children at risk of developmental coordination disorder. Human Movement Science. 2001;20(1-2):95-110.]

29. Castelnau P, Albaret JM, Chaix Y, et al. Developmental coordination disorder pertains to a deficit in perceptuo-motor synchronization independent of attentional capacities. Hum Mov Sci. 2007;26(3):477-490.)

30. Repp BH, Penel A. Rhythmic movement is attracted more strongly to auditory than to visual rhythms. Psychol Res. 2004;68(4):252-270.

31. Thaut MH. Neural basis of rhythmic timing networks in the human brain. Ann N Y Acad Sci. 2003;999:364-373.

32. Tanguy S, Quarck G, Etard O, et al. Vestibulo-ocular reflex and motion sickness in figure skaters. Eur J Appl Physiol. 2008;104(6):1031-1037.

33. Nashner LM. Computerized dynamic posturography. In: Jacobson GP, Newman CW, Artush JMK, editors. Handbook of balance function and testing. St. Louis: Mosby Yearbook Inc; 1997. p. 261-307.

34. Engel YB, Ziv OD. The relationship between sensory processing difficulties and leisure activity preference of children with different types of ADHD. Res Dev Disabil. 2011; 32(3):1154-1162.]
35. Pitcher TM, Piek JP, Barrett NC. Timing and force control in boys with attention deficit hyperactivity disorder: subtype differences and the effect of comorbid developmental coordination disorder. Hum Mov Sci. 2002;21(5-6):919-945.

36. Shum SB, Pang MY. Children with attention deficit hyperactivity disorder have impaired balance function: involvement of somatosensory, visual, and vestibular systems. J Pediatr. 2009;155(2):245-249.

37. EngelYB, Nagauker YL, Rosenblum S. Handwriting performance, selfreports, and perceived self-efficacy among children with dysgraphia. Am J Occup Ther. 2009;63(2):182-192.]

38. Jarus T, Lourie GY, Engel YB, et al. Participation patterns of school-aged children with and without DCD. Res Dev Disabil. 2011;32(4):1323-1331'

39. Engel YB, Hanna KA, Rosenblum S. The relationship between sports teachers' reports, motor performance and perceived self-efficacy of children with developmental coordination disorders. International Journal on Disability and Human Development. 2015;14: 89-96.]

40. Fong SS, Lee VY, Pang MY.Sensory organization of balance control in children with developmental coordination disorder. Res Dev Disabil. 2011;32(6):2376-2382.

41. Grove CR, Lazarus JAC. Impaired re-weighting of sensory feedback for maintenance of postural control in children with developmental coordination disorder. Hum Mov Sci. 2007;26(3):457-476.

42. Skinner RA, Piek JP. Psychosocial implications of poor motor coordination in children and adolescents. Human movement science. 2001;20(1-2):7394.

43. Missiuna C. Development of "All About Me," a scale that measures children's perceived motor competence. OTJR: Occupation, Participation and Health. 1998;18(2):85-108

44. Sturgess J, Rodger S, Ozanne A. A review of the use of self-report assessment with young children. The British Journal of Occupational Therapy. 2002;65(3):108-116.

45. Kaplan BJ, Wilson BN, Dewey D, et al. DCD may not be a discrete disorder. Human movement science. 1998;17(4-5):471-490.

46. Poulsen AA, Ziviani JM, Cuskelly M. Leisure time physical activity energy expenditure in boys with developmental coordination disorder: The role of peer relations self-concept perceptions. OTJR: Occupation, Participation and Health. 2008;28(1):30-39. 